\title{
Study of neutron-induced background hits in the CMS endcap muon system
}

\author{
Abhigyan Dasgupta*t \\ University of California, Los Angeles \\ E-mail: abhigyan.dasgupta@cern.ch
}

\begin{abstract}
Among the many challenges arising from the high luminosities of the HL-LHC is the impact of increased hit rates in the cathode strip chambers of the CMS endcap muon system. These chambers detect muons as part of the CMS muon trigger, and their data is used offline for muon track reconstruction. Neutrons (both fast and thermal) induce background hits via nuclear interactions and capture, followed by gamma emission and (mainly) Compton scatter off electrons that subsequently ionize the chamber gas. This note presents recent efforts to improve the understanding of such neutron-induced background through detailed comparison of CMS pp collision data and GEANT4 simulation.
\end{abstract}

EPS-HEP 2017, European Physical Society conference on High Energy Physics 5-12 July 2017

Venice, Italy

*Speaker.

${ }^{\dagger}$ on behalf of the CMS Muon Group 


\section{Introduction}

Proton-proton collisions and subsequent hadronic showers at the Large Hadron Collider (LHC) produce neutrons. Both fast $(\sim \mathrm{MeV})$ and thermal (sub-eV) neutrons induce background hits in the Compact Muon Solenoid (CMS) muon detectors by producing gammas via neutron capture and nuclear interactions, which subsequently lead to electrons that ionize chamber gas. Because neutrons survive a long time, these hits are delayed in time with respect to the proton-proton collisions that produced the neutrons. The impact of increased hit rates in endcap cathode strip chambers (CSCs) due to these neutron-induced hits is one of the challenges arising from the high luminosities of the High Luminosity LHC (HL-LHC).

\section{Monte Carlo Simulation of Neutrons}

Standard CMS GEANT4-based Monte Carlo simulation of proton-proton collisions [1-3] is studied, with two modifications to facilitate study of neutrons: neutrons are tracked by the simulation for 10 seconds (instead of a few hundred ns); and neutrons, protons, and ions are tracked all the way down to zero kinetic energy.

Figure 1 shows a scatter plot of the energy of neutrons just before they get captured or scatter inelastically vs. the time elapsed since the pp collision of the hit induced by the photon produced by the neutron. There are distinct classes of neutrons with different energies and flight times for which neutron interactions may produce hits in the CSCs: fast neutrons produce hits mainly by inelastic scattering, $\mathrm{keV}$ neutrons lead to resonant captures mostly on iron and copper, and finally thermal neutrons lead to more neutron captures.

The energy spectrum of the simulated photons that lead to hits ranges from $\sim 100 \mathrm{keV}$ to $\sim 10 \mathrm{MeV}$. Categorizing these photons by the specific production process that created or scattered the simulated electron responsible for the hit-either Compton scattering, pair production, or the photoelectric effect—shows that simulated hits are primary the result of Compton scattering.

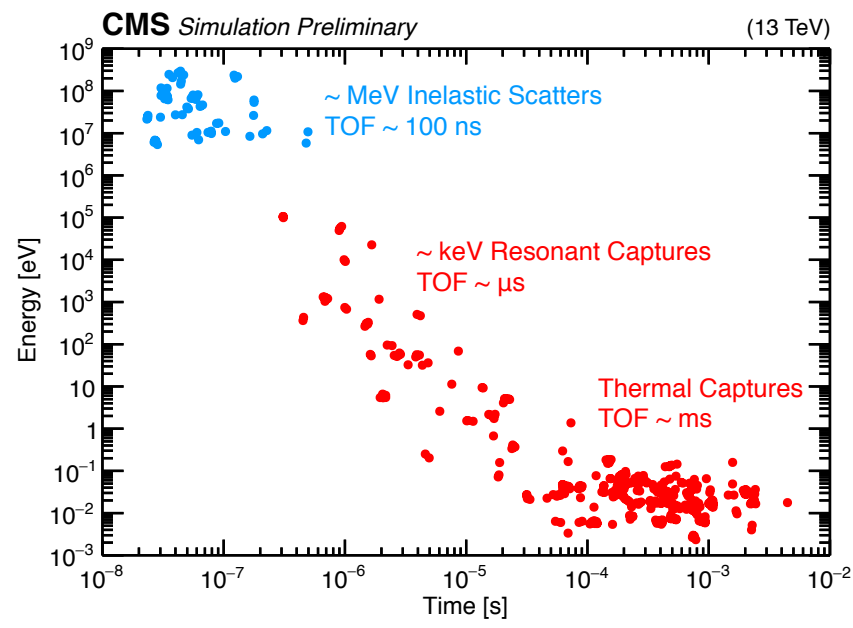

Figure 1: Simulated neutron energy just before capture or hadronic interaction vs. time since pp collision of subsequent simulated hit 


\section{Identifying Neutron Hits in CMS Data}

In CMS CSCs, a muon is required to trigger readout of local data, bringing with it associated muon-induced background [4,5]. This makes the identification of neutron-induced hits among muon-induced background in CMS data challenging. We isolate neutron-induced hits in CMS data as follows. To reduce any stray hadronic activity, we look only at $\mathrm{Z} \rightarrow \mu \mu$ events. To identify neutron hits within the chamber, we look at portions of the chamber far away from the triggering muon. We select chambers with one (and exactly one, so as not to contaminate candidate neutron hits) triggering muon in a 1/16th corner of a chamber. Then, we only consider hits that occurred in the opposite half of the chamber from this muon. Figure 2 illustrates our initial selection of neutron-induced hits.

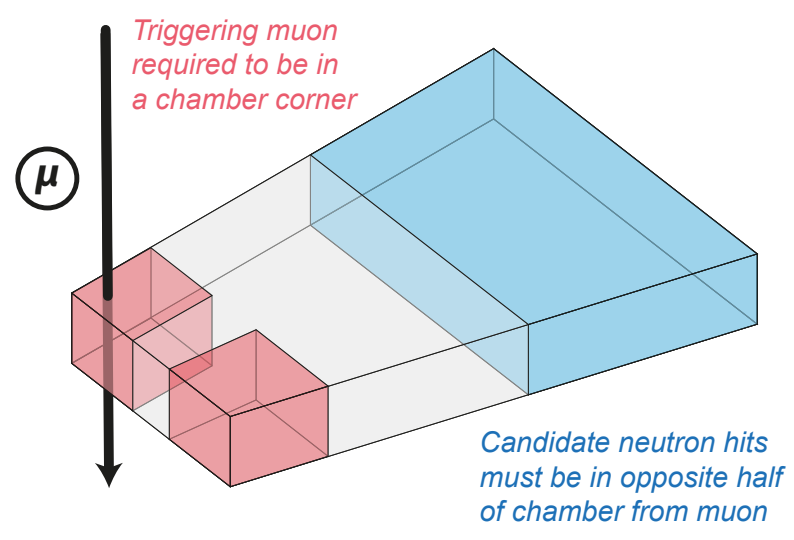

Figure 2: Diagram illustrating spatial selection of neutron hits in CSCs

To distinguish neutrons from pp collisions in earlier bunch crossings, we take advantage of gaps in the LHC proton bunch structure. The LHC proton beam has 3564 bunch places, with a bunch spacing of $25 \mathrm{~ns}$. Not every bunch place has protons in it; rather, consecutive bunches of protons occur in trains, separated by gaps [6]. Proton-proton collisions occur in CMS when bunches of protons cross, called a bunch crossing (BX). We consider trains of BX occurring after gaps of at least 35 bunch places. Anode hits are read out from CSCs in $25 \mathrm{~ns}$ time bins corresponding to each bunch place. In-time hits occur in the middle of the readout window, with 8 bunch places read out before and after, allowing us to study early-time and late-time hits [4,5]. Any early-time hits in these initial BX occur in the previous LHC gap, which means they are produced by particles created from pp collisions before the gap, i.e. hundreds of ns before the hit is recorded. These hits can therefore only have been produced by thermal or resonant neutron capture.

Figure 3 shows a 2D histogram of the CSC anode wire hit intensity (density given by color code) as a function of the number of BX after a gap ( $y$-axis) and readout time bin ( $x$-axis). Both $\mathrm{BX}$ and time bins are $25 \mathrm{~ns}$ wide. Time bin 8 is in-time with the triggering muon. The lower left blue triangle (red outline) is a region delineating early hits in early bunch places, i.e. hits recorded during an LHC gap, and contains only slow, thermal neutron hits. The excess hits in readout time bin 0 contain hits from the previous bunch crossing due to the length of electronic pulses and are therefore not considered. 


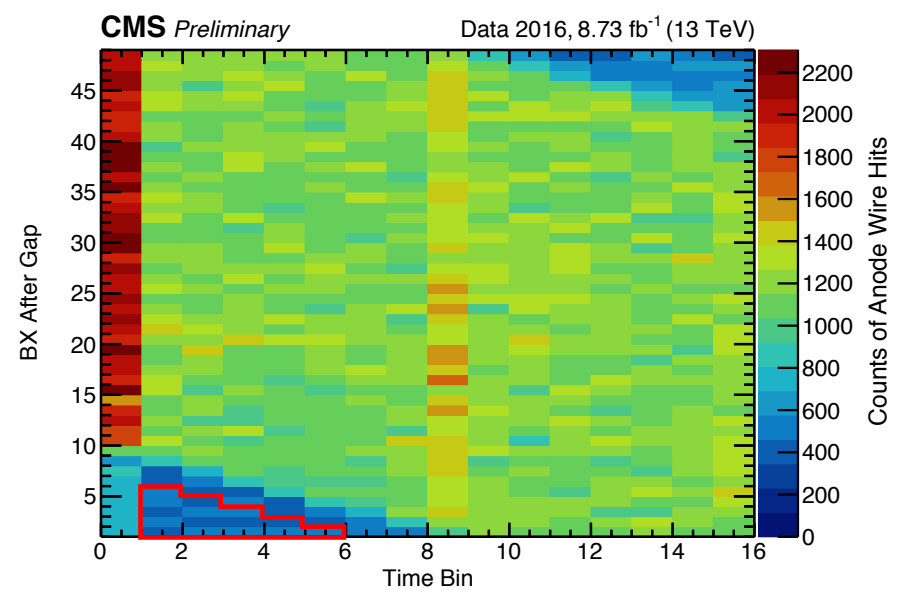

Figure 3: Plot of CSC anode wire hit intensity as a function of BX after gap and readout time bin. Time bin 8 is in-time with the triggering muon. The blue region in the lower left corner (red outline) contains early-time hits in early bunch places, which can only have occurred after a gap of 35 bunch places, and which therefore consist only of slow, thermal neutron hits.

\section{Comparing CMS Data and Simulation}

With neutron hits in CMS data thus identified, we compare isolated thermal neutron hits in CMS data with neutron hits in Monte Carlo simulation. Figure 4 shows sample distributions of candidate neutron-induced anode wire hits in CSCs from the inner ring of the second station of the CMS detector, per chamber area and converted to a per time and per pp collision rate, for CMS data and simulation. The left plot uses the High Precision (HP) nuclei cross section library containing parametrized data points, while the right plot uses XS, which refers to the less CPU-intensive approximation to HP [3]. Data were selected as described in the previous section, requiring that hits only be from the lower left triangle outlined in Figure 3. The data to simulation agreement for all $\eta$ and $z$ is within a factor of 2: the agreement depends on chamber type. With the accuracy of the Monte Carlo simulation of thermal neutrons thus quantified, studies of the potential of CMS in the HL LHC era are proceeding.
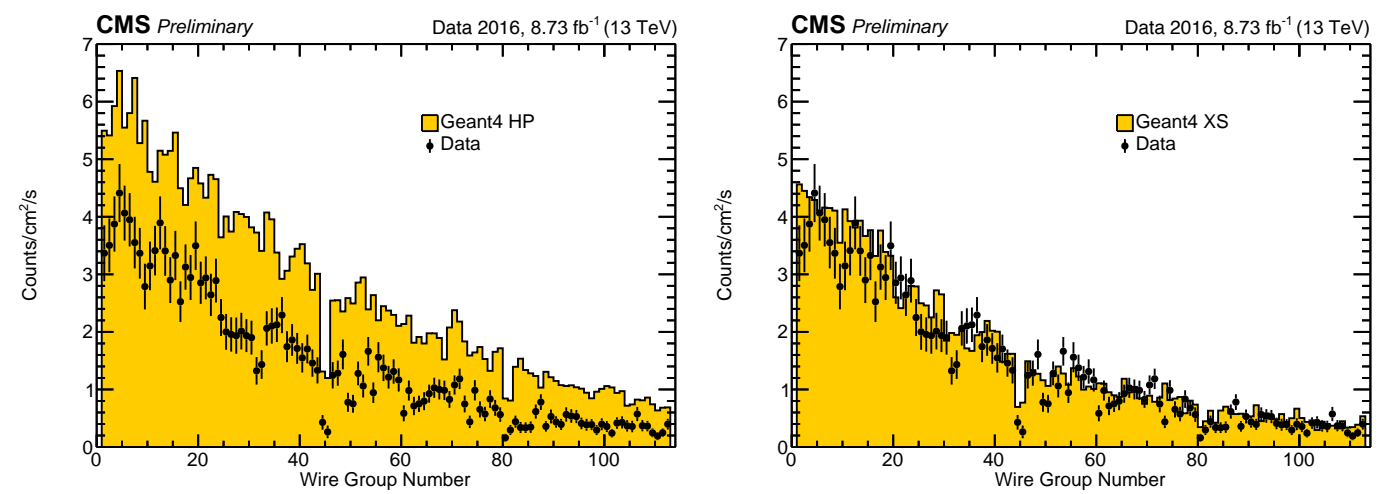

Figure 4: Sample distributions of neutron-induced anode wire hits in CSCs for CMS data and simulation, for $\mathrm{HP}$ and XS cross section libraries 


\section{References}

[1] Geant4 Collaboration, Geant4-A Simulation Toolkit, Nuclear Instruments and Methods A 506 (2003) 250, doi:10.1016/S0168-9002(03)01368-8

[2] J. Allison et al., Geant4 Development and Applications, IEEE Transactions on Nuclear Science 53 No. 1 (2006) 270, doi:10.1109/TNS.2006.869826

[3] J. Allison et al., Recent Developments in Geant4, Nuclear Instruments and Methods A 835 (2016) 186, doi:10.1016/j.nima.2016.06.125

[4] The CMS Collaboration, CMS, the Compact Muon Solenoid. Muon Technical Design Report, CERN-LHCC-97-32 (1997), see in particular pp. 169-190

[5] J. Hauser et al., Experience with Trigger Electronics for the CSC System of CMS, Proceedings of the 10th Workshop on Electronics for LHC Experiments and Future Experiments (2004)

[6] L. Evans and P. Bryant, LHC Machine, Journal of Instrumentation 3 S08001 (2008), doi:10.1088/1748-0221/3/08/S08001, see in particular pp. 138-145 